\title{
Multispectral Image Analysis approach to detect adulteration of beef and
} pork in raw meats

\author{
A.I. Ropodi ${ }^{1, a}$, D.E. Pavlidis ${ }^{1, a}$, F. Mohareb ${ }^{2}$, E.Z. Panagou ${ }^{1}$ \& G.-J.E. Nychas ${ }^{1, *}$ \\ ${ }^{1}$ Agricultural University of Athens, School of Food, Biotechnology \& Development, Dept Food Science \\ \& Human Nutrition, Lab of Microbiology \& Biotechnology of Foods, lera Odos 75, Athens 11855 \\ ${ }^{2}$ The Bioinformatics Group, Biomedical Engineering Centre, Cranfield University, College Road, \\ Bedford, MK43 OAL, UK, \\ a: These authors contributed equally to this study. \\ * Corresponding author's e-mail: gjn@aua.gr
}

\begin{abstract}
The aim of this study was to investigate the potential of multispectral imaging supported by multivariate data analysis for the detection of minced beef fraudulently substituted with pork and vice versa. Multispectral images in 18 different wavelengths of 220 meat samples in total from four independent experiments ( 55 samples per experiment) were acquired for this work. The appropriate amount of beef and pork-minced meat was mixed in order to achieve nine different proportions of adulteration and two categories of pure pork and beef. After an image processing step, data from the first three experiments were used for Partial Least Squares-Discriminant Analysis (PLS-DA) and Linear Discriminant Analysis (LDA) so as to discriminate among all adulteration classes, as well as among adulterated, pure beef and pure pork samples. Results showed very good discrimination between pure and adulterated samples, for PLS-DA and LDA, yielding $98.48 \%$ overall correct classification. Additionally, $98.48 \%$ and $96.97 \%$ of the samples were classified within a $\pm 10 \%$ category of adulteration for LDA and PLS-DA respectively. Lastly, the models were further validated using the data of the fourth experiment for independent testing, where all pure and adulterated samples were classified correctly in the case of PLS-DA, while LDA was proved to be less accurate.
\end{abstract}

\section{Keywords:}

Meat adulteration, multispectral image analysis, Discriminant Analysis, minced beef/ pork, external validation 


\section{Introduction}

Nowadays European consumers are increasingly demanding information and reassurance not only on the origin but also on the content of their food. Protecting consumer rights and preventing fraudulent or deceptive practices such as food adulteration are important and challenging issues facing the European food industry, as manufacturers are required to provide and confirm the authenticity and point of origin of food products and their components. Furthermore, adulterants can be revealed with great difficulty in the context of methods commonly applied in laboratories. Several standard analytical techniques, such as immunological and enzymatic techniques, DNA and protein based assays and triacylglycerol analysis have been applied to authenticate food commodities (Ballin, 2010; Soares, Amaral, Mafra, \& Oliveira, 2010). However, while these methods are usually capable of detecting low levels of adulteration (Ballin, 2010) they are expensive, invasive, sophisticated, laborious, and technically demanding (Ding \& Xu, 1999).

Indeed meat adulteration is a growing challenge for EU meat manufacturers since most adulterants are unknown and unpredictable (e.g., horse meat). For this reason attention should also be paid to the safety and authenticity of meat and meat products, as they can be attractive targets for adulteration in many ways, including substitution or partial substitution of high commercial value meat with cheaper, such as pork or offal or by adding proteins from several origins (Kamruzzaman, Sun, ElMasry, \& Allen, 2013; Tian, Wang, \& Cui, 2013). With minced meat being the basic ingredient for burgers, adulteration of beef minced meat is a current problem, involving economic, quality, safety and socio-religious issues (Alamprese, Casale, Sinelli, Lanteri, \& Casiraghi, 2013). Thus, the meat industry urgently needs methods that will screen non-targeted food samples for contaminants in order to provide proof of origin and prevent deliberate or accidental undeclared admixture to food samples, in a rapid and cost efficient way 
Hyperspectral and multispectral imaging spectroscopy have been used as rapid techniques to monitor quality attributes of food products (Wu \& Sun, 2013). The former has been used for the rapid detection of total viable counts in pork (Barbin, Sun, \& Su, 2013; Huang, Zhao, Chen, \& Zhang, 2013) and of the water-holding capacity of fresh beef (EIMasry, Sun, \& Allen, 2011) and pork (Prevolnik, Čandek-Potokar, \& Škorjanc, 2010). Meanwhile, multispectral image analysis has high potency for the evaluation of food quality systems during handling, processing and storage (Løkke, Seefeldt, Skov, \& Edelenbos, 2013) and it has been previously used for the conversion of meat colour in $L^{*}, a^{*}, b^{*}$ values (Sharifzadeh, Clemmensen, Borggaard, Støier, \& Ersbøll, 2014) and for quality assessment of beef (Dissing et al., 2013; Panagou, Papadopoulou, Carstensen, \& Nychas, 2014). Despite the fact that hyperspectral imaging has been used for the detection of minced lamb adulteration (Kamruzzaman et al., 2013) and gelatine adulteration in prawn (Wu, Shi, He, Yu, \& Bao, 2013), to the best of our knowledge the use of multispectral image analysis for meat adulteration, especially in the case of minced beef with pork, has never been previously explored.

Surface chemistry, such as multispectral image spectroscopy, is introduced in the present study as a new approach in tandem with advanced statistical approaches, for the discrimination of raw minced beef meat, which has been fraudulently substituted or combined with raw minced pork. Thus, the objective of this study was to (a) evaluate the potential use of multispectral imaging to discriminate pork from beef, (b) identify if possible, the lowest percentage of minced pork adulteration in minced beef that can be safely detected and (c) establish a rapid and non-invasive technique that can potentially give results in a few minutes. 


\section{Materials and Methods}

\subsection{Sample preparation}

Different levels of adulteration of minced beef and pork were prepared as follows; Fresh beef and pork fillets Longissimus muscle of normal $\mathrm{pH}(5.6-5.8)$ were purchased from central butcher shops in Athens and transported under refrigeration to the laboratory within 30 min. The fillets were cut into smaller pieces and grinded separately one at a time, using a domestic meat-mincing machine. The machine parts coming in contact with the meat were initially disinfected by washing with detergent and hot water, and rinsing with pure ethanol. To achieve different levels of adulteration, ranging from 10 to $90 \%$ with a $10 \%$ increment, the appropriate amount of each type of meat was used and mixed in conditions that simulate industrial processing. From each level of adulteration, five different portions of ca. 75-80 g were placed in Petri dishes and snapshots were taken using VideometerLab vision system (Videometer A/S, Hørsholm, Denmark). For every level of adulteration (nine categories of mixed meat and two categories of pure pork and beef), each Petri dish was considered as a replicate in the experiment ( $5 \times 11$ samples in total per experiment). All experimental procedure took place aseptically and was repeated four times. One hundred and sixty five (165) samples from three independent experiments (i.e., 55 samples per batch) were used to develop the model and 55 samples from the fourth experiment were employed for the purpose of external validation. It should be noted that 220 samples from different batches were analysed in total. From this point on, meat samples from the previously mentioned independent experiments will be referred to as samples from batches

\section{1, 2, 3 and 4.}




\subsection{Image acquisition and analysis}

Images from every sample were captured using VideometerLab, a system which acquires multispectral images in 18 -non uniformly distributed- different wavelengths ranging from 405 to $970 \mathrm{~nm}$. Analytically, the wavelengths are 405, 430, 450, 470, 505, 565, 590, 630, 645, $660,850,870,890,910,920,940,950$ and $970 \mathrm{~nm}$. The system has been developed by the Technical University of Denmark and commercialized by "Videometer A/S" (Carstensen \& Hansen, 2003; http://www.videometer.com). A detailed description of the instrument has been reported elsewhere (Panagou et al., 2014). The advantage of this instrument is that it not only uses the information of visible and short-NIR spectral regions, but moreover uses the spatial information of each pixel.

The system was first calibrated radiometrically and geometrically using well-defined standard targets, followed by a light setup based on the type of object to be recorded (FolmHansen, 1999) called "autolight". In autolight, it is always the brightest sections in the image that dictate the final result. Petri dishes (75-80 g meat portions) were placed inside an Ulbricht sphere in which the camera is top-mounted. For every random dish in each level of adulteration, a different autolight procedure was employed.

The resulting image includes redundant information, such as the Petri dish and its surrounding background, as well as the fat and connective tissue of the meat. For this reason an image-processing step is needed that will result in an image mask where only meat tissue is included. This step, which includes transformation and segmentation procedures, was implemented using the respective routines of the VideometerLab software (version 2.12.39) that controls the operation of the instrument. Canonical Discriminant Analysis (CDA) was employed as a two-step supervised transformation building method to divide the images into regions of interest (Daugaard, Adler-Nissen, \& Carstensen, 2010). Following this transformation, the separation was distinct and a simple threshold was enough to separate 
meat from non-meat pixels. The result of this processing is a segmented image for each meat sample with the isolated part of the meat tissue as the main region of interest (ROI) to be used for the extraction of spectral data that were further employed in statistical analysis. The procedure is graphically presented in Fig.1.

\subsection{Data analysis}

For each image, the mean reflectance spectrum was calculated by averaging the intensity of pixels within the ROI at each wavelength. Furthermore, the standard deviation of the pixels' intensity per wavelength was extracted. The resulting data consisted of 18 mean values and 18 standard deviations of the reflectance, as it was recorded by the camera for the pixels that were included in each image's ROI, and were further analysed with various classification methods.

Two methods, Partial Least Squares Discriminant Analysis (PLS-DA) (Barker \& Rayens, 2003; de Jong, 1993) and Linear Discriminant Analysis (LDA) (Fisher, 1936), were performed in order to discriminate among all adulteration classes (11 in total), as well as among adulterated, pure beef and pure pork samples.

As both methods are supervised, the data was partitioned in two sets: the training set used for model calibration and the test set used for validation. A $60-40 \%$ stratified partition was applied on the first three batches, meaning $60 \%$ of the dataset was chosen in a random way for calibration (99 samples out of 165) as long as all classes and batches were included and equally represented. The fourth batch was also reserved for independent model validation. Model performance was measured in terms of Recall (sensitivity) and Precision, as well as overall correct classification (OCC) (Sokolova \& Lapalme, 2009). Especially in the case of PLSDA, the optimum number of PLS components was estimated using stratified three-fold crossvalidation. 
Lastly, Hierarchical Cluster Analysis - HCA (Everitt, Landau, Leese, \& Stahl, 2011) was performed per batch as an unsupervised technique to explore the relationship between variables and adulteration classes, using Euclidean Distance and Ward's minimum variance agglomeration method. Then, Principal Component Analysis - PCA (Jolliffe, 2002) was performed per batch, as well as with all three batches so as to visualise whether there were significant differences among samples from different batches, as well as among different classes.

The partitioning algorithms of the dataset and the LDA algorithm were implemented in MATLAB R2012a (The MathWorks, Inc., Natick, Massachusetts, United States), while HCA, PCA and PLS-DA were implemented in R v.3.0.2 (RStudio, v. 0.97.551, RStudio, Inc., Boston, Massachusetts, United States), using the "plsgenomics" package (A. Boulesteix, 2004; A.-L. Boulesteix \& Strimmer, 2007; de Jong, 1993). Lastly, a heatmap was created using the MetaboAnalyst 2.0 software (Xia, Mandal, Sinelnikov, Broadhurst, \& Wishart, 2012).

\section{Results}

\subsection{Multispectral image data}

Selected spectra of minced beef in various adulteration levels are presented in Fig.2. It is characteristic that the reflectance of the sample increased in most wavelengths of the spectrum with increasing proportions of pork meat in the mixture, providing strong evidence for the effectiveness of multispectral imaging in discriminating meat adulteration. Initial analysis with HCA and PCA showed that the use of different batches is critical, as we must take into account not only the variability within a batch (different samples of the same class), but also the variability among batches. This was evident either when examining the results of HCA per batch (see Supplement File) or the heatmap for all three batches (Fig.3). When all three batches were compared, samples belonging in the same category showed 
great differences among batches. Nevertheless, a potential for good discrimination could be concluded after PCA analysis. Different Principal Components (PCs) contributed differently in terms of the variability explained (results not shown), but the first two PC scores are presented in Fig.4. In all cases, pure pork and pure beef were found on the far left and right of the plot respectively and the discrimination between classes was more evident. Only adjacent categories sometimes overlap. On the other hand, classes of mixed samples seem to be represented in a different way for each batch. Furthermore, when all three batches were included (Fig.4d), a definite trend to the right of the plot was seen as the percentage of beef in the mix increases, but the discrimination among classes was less evident. Based on the above observations, a $60-40 \%$ stratified partition was applied in the first three batches of meat corresponding to three samples per batch per category for calibration and two for validation (i.e., 99 and 66 samples in total, respectively). The samples of the fourth batch (55 in total) were reserved for independent/ external model validation.

\subsection{Validation}

The analysis of the data acquired for each class of adulteration is shown in Table 1, where the results for per-class Recall and Precision are presented. The overall correct classification, mean per-class Recall and Precision were $83.33 \%, 83.33 \%$ and $84.46 \%$ respectively, the classification error for $98.48 \%$ of the samples was at most $10 \%$, for LDA. The classification of each sample of the validation set is presented along with the per-class Precision and Recall in Table 1 in the supplement file. Very good results were also acquired when classification among pure pork, adulterated and pure beef was tested (Table 2), where the overall correct classification, mean per-class Recall and Precision was over 94\% (Mean Recall: 94.44\%, Precision: 99.39\% and overall correct classification: 98.48\%). In fact, an only one out of 66 sample was misclassified. 
Application of PLS-DA for the three-class case, yielded the same results $(98.48 \%$ correct classification) using 12 PLS components after cross-validation. PLS-DA for all categories gave similar results to the LDA. The calibration of the model was done with cross-validation, as described previously, based on overall correct classification criterion, using 20 PLS components (Table 1, and S-Table 2 in the supplement file). It would be interesting to note that, although the overall correct classification dropped considerably, this method classified $96.97 \%$ of the samples within the $\pm 10 \%$ error of prediction.

In the case of external validation, PLS-DA performed well, classifying all samples correctly in the three-class problem of pure vs. adulterated samples (Table 2). In the case of 11 categories, all pure samples were classified correctly, but the prediction of adulteration levels in the samples was less accurate (Table 1). However, only 4 out of 45 adulterated samples were classified as pure (see S-Table 4 in the supplement file).

The LDA model was less successful in predicting pure beef samples (Table 1), as well as the adulteration level for the case of 11 categories (S-Table 3 in the supplement file), whereas in the case of pure vs. adulterated samples, the results were better yielding an overall correct classification of $80 \%$ and mean per-class Recall and Precision of $85.93 \%$ and $77.08 \%$, respectively (Table 2 )

\section{Discussion}

This work investigated whether an emerging, non-destructive, fast and low cost technique (in the long term) based on imaging technology combined with multivariate data analysis can be a promising tool for the detection of minced meat adulteration. Since various standard analytical methods are now available to identify meat's adulteration at a very low level (Ballin, 2010; Ballin, Vogensen, \& Karlsson 2009), the proposed method showed great potential. In comparison with these reported methods (Ballin et al., 2009) which are time- 
consuming, expensive, use harmful reagents, need expert laboratory staff and are strongly dependent on rigorously following a standardized protocol to obtain accuracy, the multispectral imaging is a non-destructive, requires only basic training in a user-friendly software, a few minutes for image acquisition and processing and no cost at all-excluding initial instrument and software purchase.

Compared to other similar published studies on rapid techniques, in most cases, the main objective is the differentiation among different types of meat, e.g. beef vs. kangaroo (Ding \& Xu, 1999), pork vs. beef vs. lamb (Kamruzzaman, Barbin, ElMasry, Sun, \& Allen, 2012), beef vs. horsemeat (Boyacı et al., 2014). Few studies has been published on the adulteration of poultry with pork (Soares et al., 2010), pork in minced mutton (Tian et al., 2013), pork in beef meatball (Rohman et al., 2011), pork meat in raw beef burger (Giaretta, Di Giuseppe, Lippert, Parente, \& Di Maro, 2013), minced lamb (Kamruzzaman et al., 2013), gelatin in prawn (Wu et al., 2013), different types of caviar in Caspian caviar (Mohamadi Monavar et al., 2013). Some have been reported in the case of classification between adulterated and pure samples with different percentages of adulteration (Alamprese et al., 2013; Kamruzzaman et al., 2013; Tian et al., 2013), and the case of beef adulterated with pork (Morsy \& Sun, 2013; Rohman et al., 2011). In this study, pure beef, pure pork and nine levels of adulteration were employed in order not only to discriminate but also to quantify the minimum possible level of adulteration detected.

As in most of the above cases, HCA, PCA, LDA and PLS-DA were the predominant methods used for data analysis. Results showed that multispectral imaging has the potential to identify adulterated beef samples with pork and vice versa in a rapid, non-invasive way. Furthermore, the variability between meat batches was taken into account - an important issue that is not always presented in the available literature- by using three different batches for model training and testing, and a fourth external batch for validation. Results showed 
that a $10 \%$ adulteration with pork in beef and vice versa could be successfully identified and could thus be considered as a detection limit of the applied method, which can be related to the results by Morsy and Sun (Morsy \& Sun, 2013) using NIR spectroscopy, although no external validation was performed in this work, and the results by Alamprese et al.(2013), in the case of adulteration with turkey.

It should be noted that very few of the abovementioned studies use external batch validation, results demonstrated -especially for LDA- the necessity of such an approach in order to exclude cases of overoptimistic results. On the other hand the quantification of the level of adulteration was proved to be more difficult task. A large number of adulteration classes were used (11 classes in total), whereas in other studies discriminant analysis was performed with fewer categories. For example, Alamprese et al. (2013) used 5 classes for cross-validation, grouping very low adulteration with pure samples. However, in this study the applied method was found to provide additional information on the detection limit of $10 \%$ and as such can be considered as an advantage even if the quantification per se is of great importance.

In conclusion, multispectral imaging was used for the first time as a rapid method for food authentication and detection of adulteration of raw meat, illustrating a clear separation of pure vs. adulterated samples. PLS-DA performed better compared to LDA in the case of external batch validation. Moreover, the quantification of the percentage of adulteration was proved to be more challenging. The applied method managed to detect relatively small percentages of adulteration $(10 \% \mathrm{w} / \mathrm{w})$ of pure beef with pork and vice versa. It is therefore evident that multispectral imaging could be used as a rapid, non-invasive method for the detection of adulteration. 


\section{Acknowledgements}

This work has been supported by the project "Intelligent multi-sensor system for meat analysis -iMeatSense550" co-financed by the European Union (European Social Fund - ESF) and Greek national funds through the Operational Program "Education and Lifelong Learning" of the National Strategic Reference Framework (NSRF) - Research Funding Program: ARISTEIA-I 


\section{References}

Alamprese, C., Casale, M., Sinelli, N., Lanteri, S., \& Casiraghi, E. (2013). Detection of minced beef adulteration with turkey meat by UV-vis, NIR and MIR spectroscopy. LWT - Food Science and Technology, 53(1), 225-232. doi:10.1016/j.Iwt.2013.01.027

Ammor, M. S., Argyri, A., \& Nychas, G.-J. E. (2009). Rapid monitoring of the spoilage of minced beef stored under conventionally and active packaging conditions using Fourier transform infrared spectroscopy in tandem with chemometrics. Meat Science, 81(3), 507-514. doi:10.1016/j.meatsci.2008.10.015

Argyri, A. A., Jarvis, R. M., Wedge, D., Xu, Y., Panagou, E. Z., Goodacre, R., \& Nychas, G.-J. E. (2013). A comparison of Raman and FT-IR spectroscopy for the prediction of meat spoilage. Food Control, 29(2), 461-470. doi:10.1016/j.foodcont.2012.05.040

Ballin, N. Z. (2010). Authentication of meat and meat products. Meat Science, 86(3), 577-87. doi:10.1016/j.meatsci.2010.06.001

Ballin, N. Z., Vogensen, F. K., \& Karlsson, A. H. (2009). Species determination - Can we detect and quantify meat adulteration? Meat Science, 83(2), 165-74. doi:10.1016/j.meatsci.2009.06.003

Barbin, D. F., Sun, D.-W., \& Su, C. (2013). NIR hyperspectral imaging as non-destructive evaluation tool for the recognition of fresh and frozen-thawed porcine longissimus dorsi muscles. Innovative Food Science \& Emerging Technologies, 18, 226-236. doi:10.1016/j.ifset.2012.12.011

Barker, M., \& Rayens, W. (2003). Partial least squares for discrimination. Journal of Chemometrics, 17(3), 166-173. doi:10.1002/cem.785

Boulesteix, A. (2004). PLS dimension reduction for classification with microarray data. Statistical Applications in Genetics and Molecular Biology, 3(14), Article33. doi:10.2202/1544-6115.1075

Boulesteix, A.-L., \& Strimmer, K. (2007). Partial least squares: a versatile tool for the analysis of high-dimensional genomic data. Briefings in Bioinformatics, 8(1), 32-44. doi:10.1093/bib/bbl016

Boyacı, İ. H., Temiz, H. T., Uysal, R. S., Velioğlu, H. M., Yadegari, R. J., \& Rishkan, M. M. (2014). A novel method for discrimination of beef and horsemeat using Raman spectroscopy. Food Chemistry, 148, 37-41. doi:10.1016/j.foodchem.2013.10.006

Carstensen, J. M., \& Hansen, J. F. (2003). An apparatus and a method of recording an image of an object, Patent family EP1051660.

Dai, Q., Sun, D.-W., Xiong, Z., Cheng, J.-H., \& Zeng, X.-A. (2014). Recent Advances in Data Mining Techniques and Their Applications in Hyperspectral Image Processing for the Food Industry. Comprehensive Reviews in Food Science and Food Safety, 13(5), 891905. doi:10.1111/1541-4337.12088 
Daugaard, S. B., Adler-Nissen, J., \& Carstensen, J. M. (2010). New vision technology for multidimensional quality monitoring of continuous frying of meat. Food Control, 21(5), 626-632. doi:10.1016/j.foodcont.2009.09.007

De Jong, S. (1993). SIMPLS: An alternative approach to partial least squares regression. Chemometrics and Intelligent Laboratory Systems, 18(3), 251-263. doi:10.1016/01697439(93)85002-X

Ding, H. B., \& Xu, R. J. (1999). Differentiation of beef and kangaroo meat by visible/nearinfrared reflectance spectroscopy. Journal of Food Science, 64(5), 814-817. doi:10.1111/j.1365-2621.1999.tb15918.x

Dissing, B. S., Papadopoulou, O. S., Tassou, C., Ersbøll, B. K., Carstensen, J. M., Panagou, E. Z., \& Nychas, G.-J. (2013). Using Multispectral Imaging for Spoilage Detection of Pork Meat. Food and Bioprocess Technology, 6(9), 2268-2279. doi:10.1007/s11947-012-0886-6

Ebrahim, H. Al, Sowoidnich, K., \& Kronfeldt, H.-D. (2013). Raman spectroscopic differentiation of beef and horse meat using a $671 \mathrm{~nm}$ microsystem diode laser. Applied Physics B, 113(2), 159-163. doi:10.1007/s00340-013-5677-x

Ellis, D. I., Broadhurst, D., Clarke, S. J., \& Goodacre, R. (2005). Rapid identification of closely related muscle foods by vibrational spectroscopy and machine learning. The Analyst, 130(12), 1648-54. doi:10.1039/b511484e

ElMasry, G., Sun, D.-W., \& Allen, P. (2011). Non-destructive determination of water-holding capacity in fresh beef by using NIR hyperspectral imaging. Food Research International, 44(9), 2624-2633. doi:10.1016/j.foodres.2011.05.001

Everitt, B., Landau, S., Leese, M., \& Stahl, D. (2011). Cluster analysis (5th ed.). Wiley.

Fisher, R. A. (1936). The Use of Multiple Measurements in Taxonomic Problems. Annals of Eugenics, 7(2), 179-188. doi:10.1111/j.1469-1809.1936.tb02137.x

Folm-Hansen, J. (1999). On Chromatic and Geometrical Calibration (Ph.D. thesis). Technical University of Denmark (DTU), Department of Mathematical Modeling.

Giaretta, N., Di Giuseppe, A. M. A., Lippert, M., Parente, A., \& Di Maro, A. (2013). Myoglobin as marker in meat adulteration: a UPLC method for determining the presence of pork meat in raw beef burger. Food Chemistry, 141(3), 1814-20. doi:10.1016/j.foodchem.2013.04.124

Hastie, T., Tibshirani, R., \& Friedman, J. (2009). The Elements of Statistical Learning (2nd ed.). Springer New York.

Huang, L., Zhao, J., Chen, Q., \& Zhang, Y. (2013). Rapid detection of total viable count (TVC) in pork meat by hyperspectral imaging. Food Research International, 54(1), 821-828. doi:10.1016/j.foodres.2013.08.011

Jolliffe, I. T. (2002). Principal Component Analysis (2nd ed.). Springer New York. doi:10.1007/b98835 
Kamruzzaman, M., Barbin, D., ElMasry, G., Sun, D.-W., \& Allen, P. (2012). Potential of hyperspectral imaging and pattern recognition for categorization and authentication of red meat. Innovative Food Science \& Emerging Technologies, 16, 316-325. doi:10.1016/j.ifset.2012.07.007

Kamruzzaman, M., Sun, D.-W., ElMasry, G., \& Allen, P. (2013). Fast detection and visualization of minced lamb meat adulteration using NIR hyperspectral imaging and multivariate image analysis. Talanta, 103, 130-6. doi:10.1016/j.talanta.2012.10.020

Løkke, M. M., Seefeldt, H. F., Skov, T., \& Edelenbos, M. (2013). Color and textural quality of packaged wild rocket measured by multispectral imaging. Postharvest Biology and Technology, 75, 86-95. doi:10.1016/j.postharvbio.2012.06.018

MATLAB 2012a, The MathWorks, Inc., Natick, Massachusetts, United States. (n.d.). The MathWorks, Inc., Natick, Massachusetts, United States.

Mohamadi Monavar, H., Afseth, N. K., Lozano, J., Alimardani, R., Omid, M., \& Wold, J. P. (2013). Determining quality of caviar from Caspian Sea based on Raman spectroscopy and using artificial neural networks. Talanta, 111, 98-104. doi:10.1016/j.talanta.2013.02.046

Moore, J. C., Spink, J., \& Lipp, M. (2012). Development and Application of a Database of Food Ingredient Fraud and Economically Motivated Adulteration from 1980 to 2010. Journal of Food Science, 77(4), R118-R126. doi:10.1111/j.1750-3841.2012.02657.x

Morsy, N., \& Sun, D.-W. (2013). Robust linear and non-linear models of NIR spectroscopy for detection and quantification of adulterants in fresh and frozen-thawed minced beef. Meat Science, 93(2), 292-302. doi:10.1016/j.meatsci.2012.09.005

Nunes, C. A. (2013). Vibrational spectroscopy and chemometrics to assess authenticity, adulteration and intrinsic quality parameters of edible oils and fats. Food Research International. doi:10.1016/j.foodres.2013.08.041

Oliveri, P., \& Downey, G. (2012). Multivariate class modeling for the verification of foodauthenticity claims. TrAC Trends in Analytical Chemistry, 35, 74-86. doi:10.1016/j.trac.2012.02.005

Panagou, E. Z., Papadopoulou, O., Carstensen, J. M., \& Nychas, G.-J. E. (2014). Potential of multispectral imaging technology for rapid and non-destructive determination of the microbiological quality of beef filets during aerobic storage. International Journal of Food Microbiology, 174, 1-11. doi:10.1016/j.ijfoodmicro.2013.12.026

Papadopoulou, O., Panagou, E. Z., Tassou, C. C., \& Nychas, G.-J. E. (2011). Contribution of Fourier transform infrared (FTIR) spectroscopy data on the quantitative determination of minced pork meat spoilage. Food Research International, 44(10), 3264-3271. doi:10.1016/j.foodres.2011.09.012

Prevolnik, M., Čandek-Potokar, M., \& Škorjanc, D. (2010). Predicting pork water-holding capacity with NIR spectroscopy in relation to different reference methods. Journal of Food Engineering, 98(3), 347-352. doi:10.1016/j.jfoodeng.2009.11.022 
Rodriguez-Saona, L. E., \& Allendorf, M. E. (2011). Use of FTIR for Rapid Authentication and Detection of Adulteration of Food. Annual Review of Food Science and Technology, 2(1), 467-483. doi:10.1146/annurev-food-022510-133750

Rohman, A., Sismindari, Erwanto, Y., \& Che Man, Y. B. (2011). Analysis of pork adulteration in beef meatball using Fourier transform infrared (FTIR) spectroscopy. Meat Science, 88(1), 91-5. doi:10.1016/j.meatsci.2010.12.007

RStudio, v. 0.97.551, RStudio, Inc., Boston, Massachusetts, United States. (n.d.).

Sharifzadeh, S., Clemmensen, L. H., Borggaard, C., Støier, S., \& Ersbøll, B. K. (2014). Supervised feature selection for linear and non-linear regression of $\mathrm{L}^{*} \mathrm{a} \mathrm{b}^{*}$ color from multispectral images of meat. Engineering Applications of Artificial Intelligence, 27, 211-227. doi:10.1016/j.engappai.2013.09.004

Soares, S., Amaral, J. S., Mafra, I., \& Oliveira, M. B. P. P. (2010). Quantitative detection of poultry meat adulteration with pork by a duplex PCR assay. Meat Science, 85(3), 531-6. doi:10.1016/j.meatsci.2010.03.001

Sokolova, M., \& Lapalme, G. (2009). A systematic analysis of performance measures for classification tasks. Information Processing \& Management, 45(4), 427-437. doi:10.1016/j.ipm.2009.03.002

Sun, D. W. (2009). Infrared Spectroscopy for Food Quality Analysis and Control. (D. W. Sun, Ed.)Infrared Spectroscopy for Food Quality Analysis and Control (1st ed.). Elsevier Inc.

Tian, X., Wang, J., \& Cui, S. (2013). Analysis of pork adulteration in minced mutton using electronic nose of metal oxide sensors. Journal of Food Engineering, 119(4), 744-749. doi:10.1016/j.jfoodeng.2013.07.004

Videometer A/S (official website). (n.d.). Retrieved August 29, 2014, from http://www.videometer.com

Wu, D., Shi, H., He, Y., Yu, X., \& Bao, Y. (2013). Potential of hyperspectral imaging and multivariate analysis for rapid and non-invasive detection of gelatin adulteration in prawn. Journal of Food Engineering, 119(3), 680-686. doi:10.1016/j.jfoodeng.2013.06.039

Wu, D., \& Sun, D.-W. (2013). Advanced applications of hyperspectral imaging technology for food quality and safety analysis and assessment: A review - Part II: Applications. Innovative Food Science \& Emerging Technologies, 19, 15-28. doi:10.1016/j.ifset.2013.04.016

Xia, J., Mandal, R., Sinelnikov, I. V, Broadhurst, D., \& Wishart, D. S. (2012). MetaboAnalyst 2.0: a comprehensive server for metabolomic data analysis. Nucleic Acids Research, 40(Web Server issue), W127-33. doi:10.1093/nar/gks374

Zhao, M., Downey, G., \& O'Donnell, C. P. (2014). Detection of adulteration in fresh and frozen beefburger products by beef offal using mid-infrared ATR spectroscopy and 
multivariate data analysis. Meat Science, 96(2 Pt A), 1003-11.

doi:10.1016/j.meatsci.2013.10.015 


\section{List of figures}

Figure1. Process of multispectral image acquisition and extraction of data

Figure 2. Selected spectra of the examined samples corresponding to different ratios of adulteration

Figure 3. Heatmap of all samples from batches 1-3, different colours on the left side correspond to different ratios of adulteration. Samples named with " 00 " correspond to pure pork, all other categories correspond to the percentage of beef in the mix and consequently " 100 " refers to pure beef samples, whereas "b1", "b2", "b3" correspond to the number of batch.

Figure 4. Principal Component Analysis scores for Batches 1(a), 2(b), 3(c) and all batches (d). Samples named with "0" correspond to pure pork, all other categories correspond to the percentage of beef in the mix and consequently " 100 " refers to pure beef samples. 
Fig.1

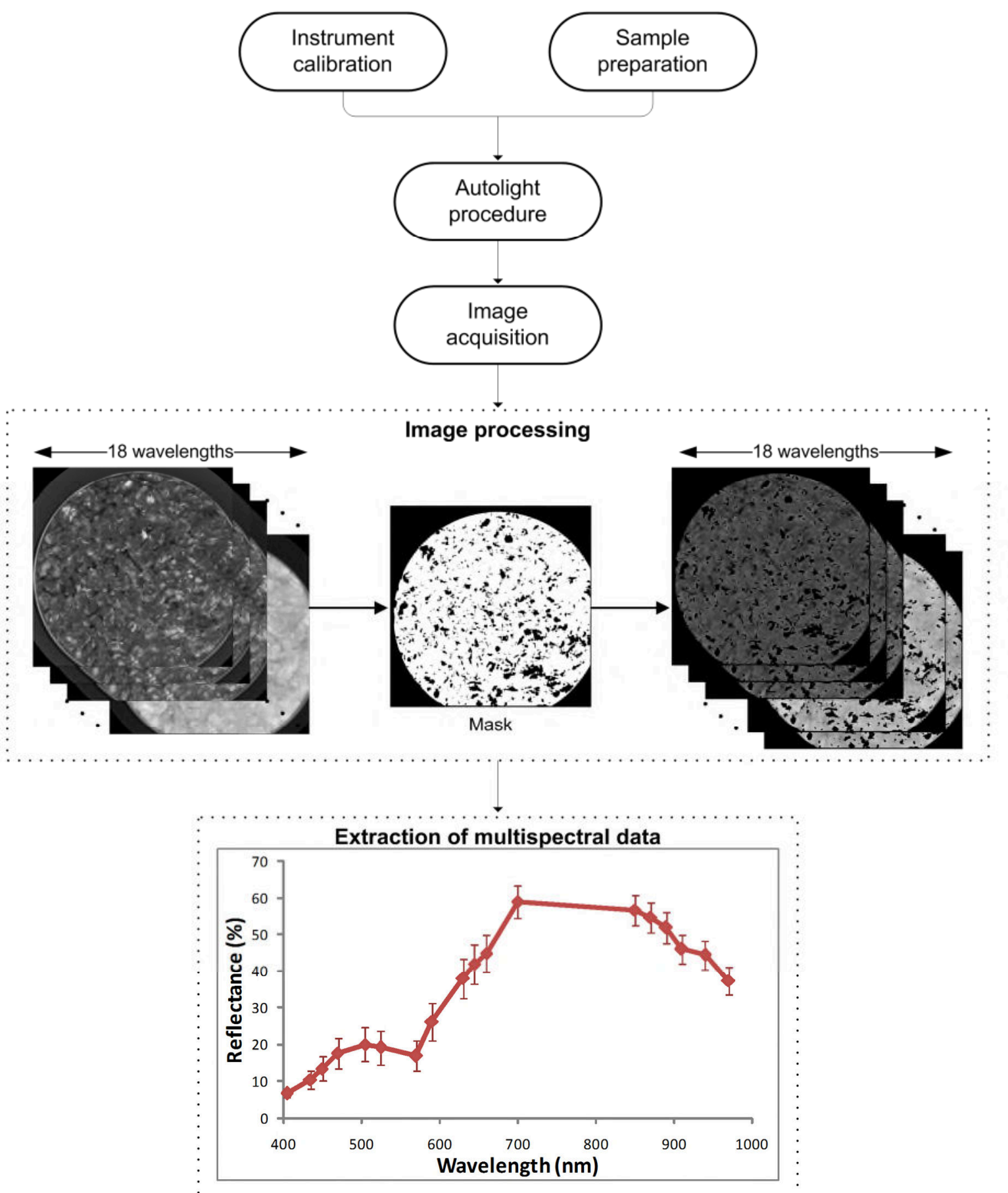




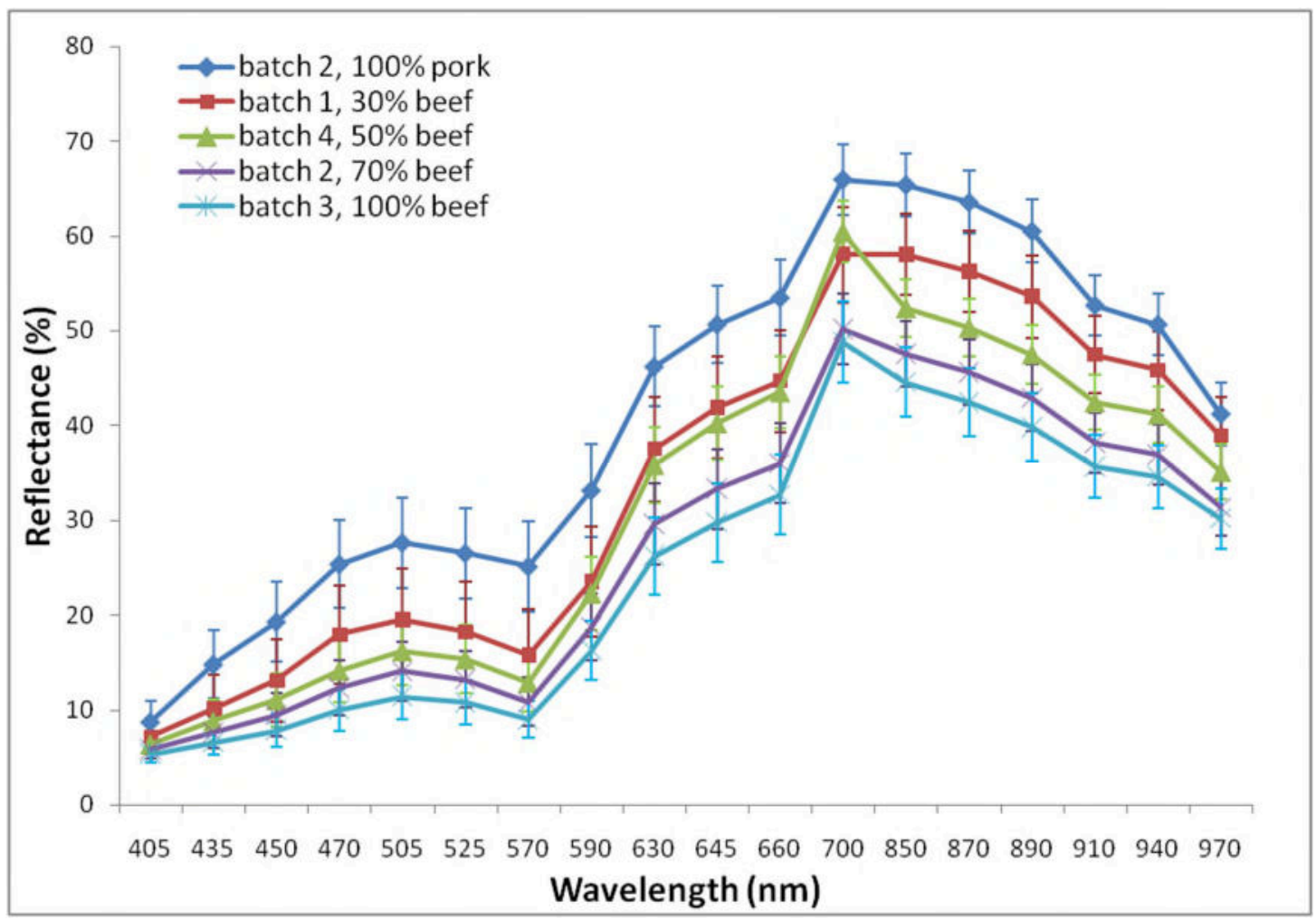

Fig. 2 


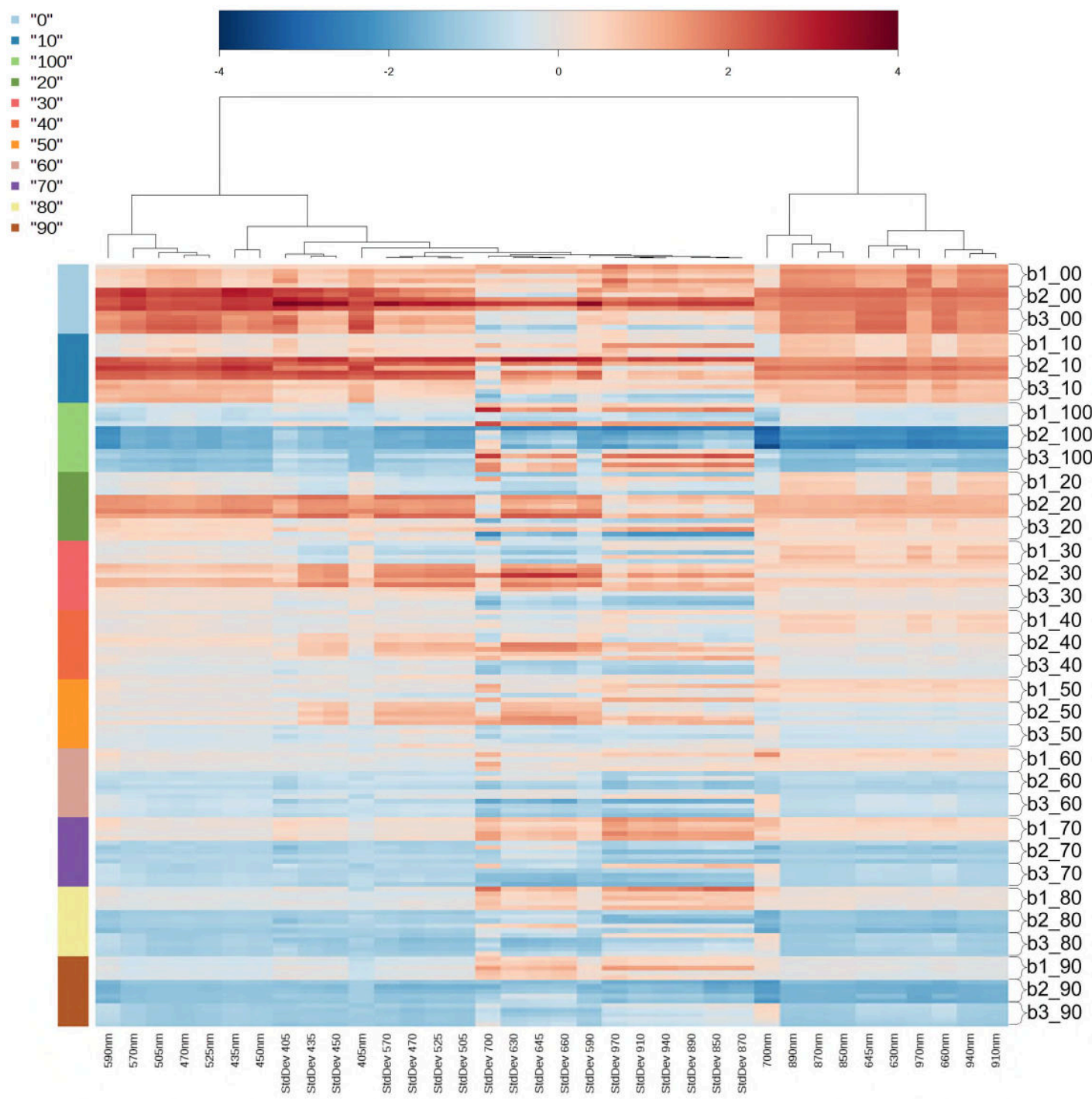

Fig. 3 

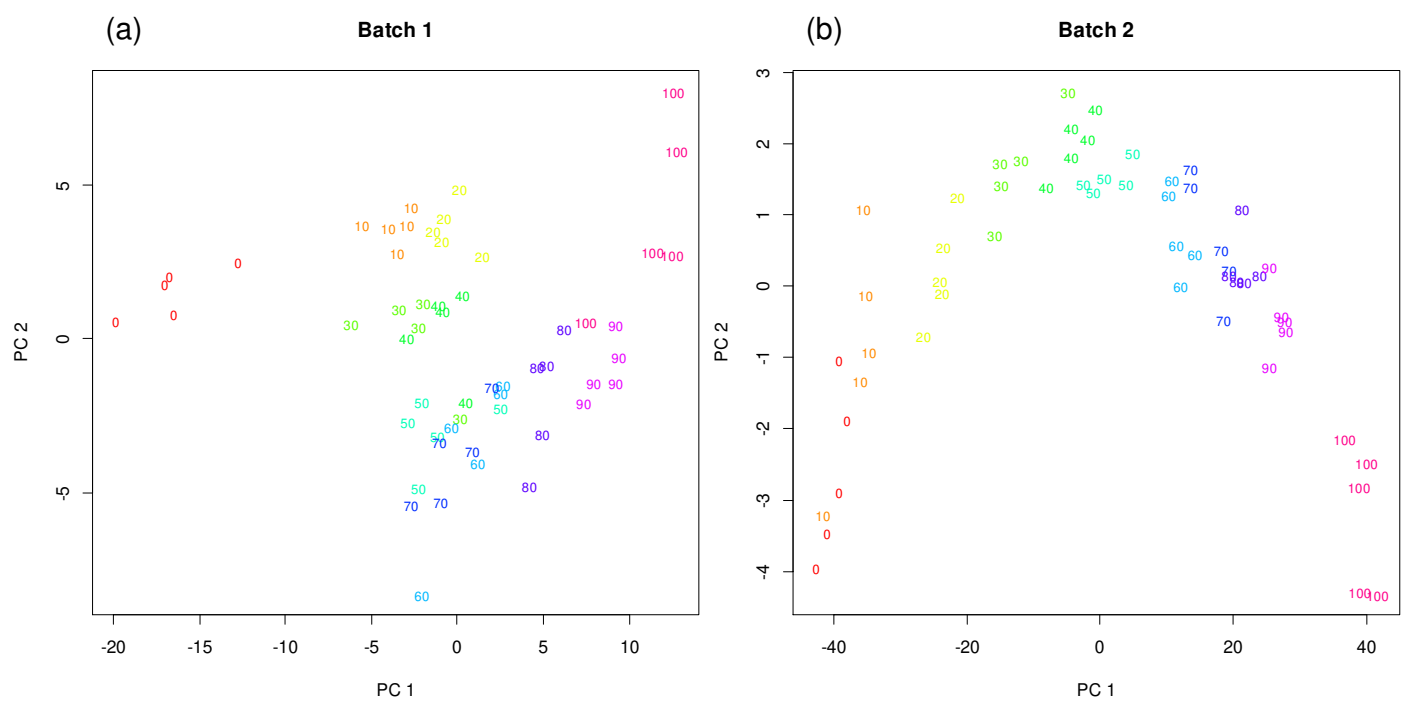

(c)

Batch 3

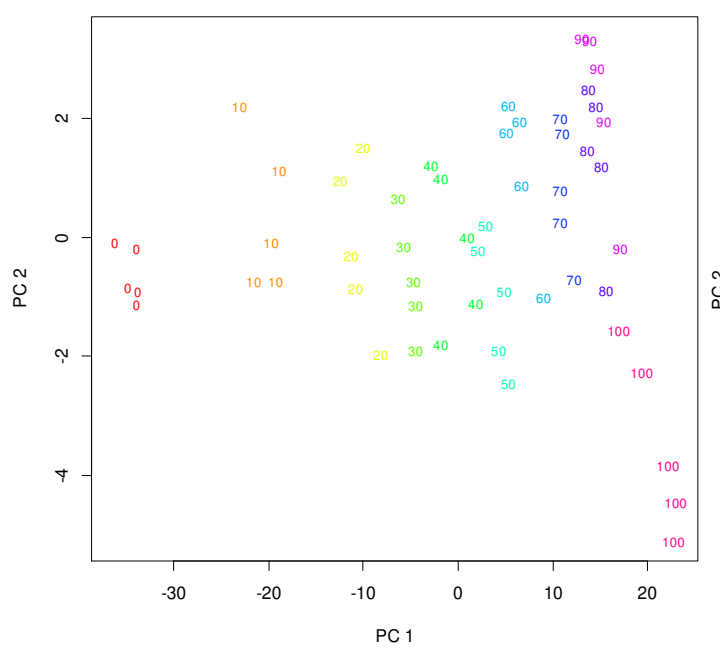

(d)

Batches 1,2 \& 3

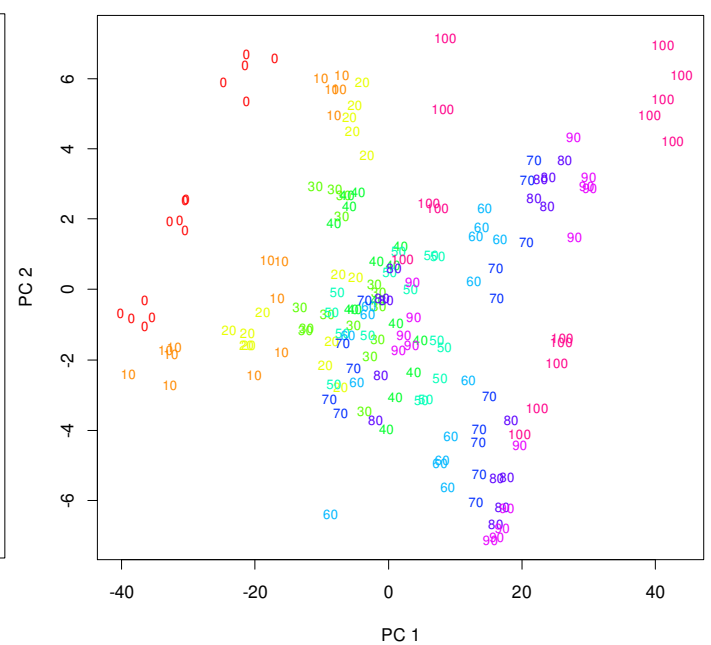

Fig. 4 
Table 1. LDA vs. PLS-DA for both validation set and external validation batch with 11 classes ranging from pure pork (0\%) to beef (100\%)

\begin{tabular}{|c|c|c|c|c|c|c|c|c|c|c|c|c|}
\hline \multirow[b]{3}{*}{ Sample } & \multicolumn{6}{|c|}{ Validation set } & \multicolumn{6}{|c|}{ External validation set } \\
\hline & \multicolumn{3}{|c|}{ LDA } & \multicolumn{3}{|c|}{ PLS-DA } & \multicolumn{3}{|c|}{ LDA } & \multicolumn{3}{|c|}{ PLS-DA } \\
\hline & Recall & Precision & $\begin{array}{l} \pm 10 \% \\
\text { error }\end{array}$ & Recall & Precision & $\begin{array}{l} \pm 10 \% \\
\text { error }\end{array}$ & Recall & Precision & $\begin{array}{l} \pm 10 \% \\
\text { error }\end{array}$ & Recall & Precision & $\begin{array}{l} \pm 10 \% \\
\text { error }\end{array}$ \\
\hline Is $0 \%$ & $83.33 \%$ & $100.00 \%$ & $100.00 \%$ & $66.67 \%$ & $100.00 \%$ & $100.00 \%$ & $100.00 \%$ & $100.00 \%$ & $100.00 \%$ & $100.00 \%$ & $100.00 \%$ & $100.00 \%$ \\
\hline Is $10 \%$ & $100.00 \%$ & $85.71 \%$ & $100.00 \%$ & $100.00 \%$ & $75.00 \%$ & $100.00 \%$ & $20.00 \%$ & $16.67 \%$ & $100.00 \%$ & $0.00 \%$ & $0.00 \%$ & $20.00 \%$ \\
\hline Is $20 \%$ & $83.33 \%$ & $100.00 \%$ & $100.00 \%$ & $83.33 \%$ & $100.00 \%$ & $100.00 \%$ & $20.00 \%$ & $7.14 \%$ & $20.00 \%$ & $0.00 \%$ & $0.00 \%$ & $20.00 \%$ \\
\hline Is $30 \%$ & $83.33 \%$ & $71.43 \%$ & $83.33 \%$ & $50.00 \%$ & $75.00 \%$ & $100.00 \%$ & $0.00 \%$ & $0.00 \%$ & $100.00 \%$ & $0.00 \%$ & $0.00 \%$ & $40.00 \%$ \\
\hline Is $40 \%$ & $66.67 \%$ & $80.00 \%$ & $100.00 \%$ & $66.67 \%$ & $50.00 \%$ & $100.00 \%$ & $20.00 \%$ & $7.69 \%$ & $80.00 \%$ & $20.00 \%$ & $14.29 \%$ & $20.00 \%$ \\
\hline Is $50 \%$ & $83.33 \%$ & $71.43 \%$ & $100.00 \%$ & $100.00 \%$ & $75.00 \%$ & $100.00 \%$ & $0.00 \%$ & $0.00 \%$ & $0.00 \%$ & $0.00 \%$ & $0.00 \%$ & $60.00 \%$ \\
\hline Is $60 \%$ & $66.67 \%$ & $80.00 \%$ & $100.00 \%$ & $33.33 \%$ & $50.00 \%$ & $83.33 \%$ & $0.00 \%$ & $0.00 \%$ & $0.00 \%$ & $0.00 \%$ & $0.00 \%$ & $0.00 \%$ \\
\hline Is $70 \%$ & $66.67 \%$ & $57.14 \%$ & $100.00 \%$ & $83.33 \%$ & $50.00 \%$ & $100.00 \%$ & $0.00 \%$ & $0.00 \%$ & $0.00 \%$ & $0.00 \%$ & $0.00 \%$ & $0.00 \%$ \\
\hline Is $80 \%$ & $83.33 \%$ & $83.33 \%$ & $100.00 \%$ & $16.67 \%$ & $100.00 \%$ & $83.33 \%$ & $0.00 \%$ & $0.00 \%$ & $0.00 \%$ & $0.00 \%$ & $0.00 \%$ & $20.00 \%$ \\
\hline Is $90 \%$ & $100.00 \%$ & $100.00 \%$ & $100.00 \%$ & $83.33 \%$ & $71.43 \%$ & $100.00 \%$ & $0.00 \%$ & $0.00 \%$ & $0.00 \%$ & $100.00 \%$ & $33.33 \%$ & $100.00 \%$ \\
\hline Is $100 \%$ & $100.00 \%$ & $100.00 \%$ & $100.00 \%$ & $100.00 \%$ & $85.71 \%$ & $100.00 \%$ & $0.00 \%$ & $0.00 \%$ & $0.00 \%$ & $100.00 \%$ & $55.56 \%$ & $100.00 \%$ \\
\hline $\begin{array}{l}\text { Mean } \\
\text { per-class }\end{array}$ & $83.33 \%$ & $84.46 \%$ & $98.48 \%$ & $71.21 \%$ & $75.65 \%$ & $96.97 \%$ & $14.55 \%$ & $11.95 \%$ & $36.36 \%$ & $29.09 \%$ & $18.47 \%$ & $43.64 \%$ \\
\hline
\end{tabular}


Table 2. LDA and PLS-DA (12 PLS components) for both validation set and external validation batch with 3 classes (pork - adulterated - beef)

\begin{tabular}{|c|c|c|c|c|c|c|c|c|c|}
\hline \multicolumn{5}{|c|}{ LDA } & \multicolumn{5}{|c|}{ PLS-DA } \\
\hline \multicolumn{10}{|c|}{ Validation set } \\
\hline & pork & $\begin{array}{l}\text { classified as } \\
\text { adulterated }\end{array}$ & beef & Recall & & pork & $\begin{array}{l}\text { classified as } \\
\text { adulterated }\end{array}$ & beef & Recall \\
\hline is pork & 5 & 1 & 0 & $83.33 \%$ & is pork & 5 & 1 & 0 & $83.33 \%$ \\
\hline is adulterated & 0 & 54 & 0 & $100.00 \%$ & is adulterated & 0 & 54 & 0 & $100.00 \%$ \\
\hline is beef & 0 & 0 & 6 & $100.00 \%$ & is beef & 0 & 0 & 6 & $100.00 \%$ \\
\hline Precision & $100.00 \%$ & $98.18 \%$ & $100.00 \%$ & & Precision & $100.00 \%$ & $98.18 \%$ & $100.00 \%$ & \\
\hline \multicolumn{10}{|c|}{ External validation Batch } \\
\hline & pork & $\begin{array}{l}\text { classified as } \\
\text { adulterated }\end{array}$ & beef & Recall & & pork & $\begin{array}{l}\text { classified as } \\
\text { adulterated }\end{array}$ & beef & Recall \\
\hline is pork & 4 & 0 & 1 & $80.00 \%$ & is pork & 5 & 0 & 0 & $100.00 \%$ \\
\hline is adulterated & 0 & 35 & 10 & $77.78 \%$ & is adulterated & 0 & 45 & 0 & $100.00 \%$ \\
\hline is beef & 0 & 0 & 5 & $100.00 \%$ & is beef & 0 & 0 & 5 & $100.00 \%$ \\
\hline Precision & $100.00 \%$ & $100.00 \%$ & $31.25 \%$ & & Precision & $100.00 \%$ & $100.00 \%$ & $100.00 \%$ & \\
\hline
\end{tabular}

